Werbena Aguiar dos Santos 1 Bethania Moraes da Silva 1 Elane Dellacqua Passos 1 Eliana Zandonade 1 Aloísio Falqueto 1

\section{Associação entre tabagismo e paracoccidioidomicose: um estudo de caso-controle no Estado do Espírito Santo, Brasil}

\author{
Association between smoking and \\ paracoccidioidomycosis: a case-control \\ study in the State of Espírito Santo, Brazil
}

1 Programa de

Pós-Graduação em Atenção

à Saúde Coletiva,

Centro Biomédico,

Universidade Federal

do Espírito Santo.

Av. Marechal Campos 1468

Vitória, ES

29040-090, Brasil.

falqueto@npd.ufes.br

werbena@terra.com.br

\begin{abstract}
Paracoccidioidomycosis, the main systemic mycosis in Brazil, requires long-term, high-cost treatment and leaves serious sequelae in the lungs, the organ most frequently affected and further subject to aggressive external risk factors like smoking. The influence of tobacco and alcohol consumption on chronic paracoccidioidomycosis was investigated using a case-control study. Data on occupation, place of residence, and living habits were obtained from 70 cases and 180 controls residing in the same geographic areas. The risk of becoming ill was 14 times greater among smokers and 3.6 times greater among individuals with an alcohol intake of more than 50g/day. Logistic regression showed as significant variables: tobacco consumption for more than 20 years $(O R=10.1)$, smoking manufactured (not hand-rolled) cigarettes $(O R=4.8)$, and alcohol intake $>50 \mathrm{~g} /$ day $(\mathrm{OR}=2.9)$. Cases who smoked 20 or more cigarettes/day became ill on average eight years before others $(p=0.002)$. Alcohol intake $>50 \mathrm{~g} /$ day had no statistically significant impact on age at onset of illness ( $p=0.78$ ). The study concludes that smoking stands as an important risk factor for the development of chronic paracoccidioidomycosis. As for alcoholism, there is evidence that it acts as a co-factor, together with smoking.
\end{abstract}

Key words Paracoccidioidomycosis; Smoking; Alcoholism; Risk Factors

Resumo A paracoccidioidomicose é a principal micose sistêmica em nosso meio, que exige tratamento prolongado, de alto custo, e que deixa seqüelas graves nos pulmões, órgãos mais freqüentemente acometidos e sujeitos ainda às agressões de fatores de risco externos, como o tabaco. Investigou-se, por meio de um estudo caso-controle, a influência do tabagismo e do alcoolismo no desenvolvimento da forma crônica da paracoccidioidomicose. Dados sobre a ocupação, moradia e hábitos de vida foram obtidos de 70 doentes e 180 controles. Os doentes fumavam e bebiam mais que os controles. A chance de adoecer foi 14 vezes maior entre os fumantes e 3,6 vezes maior entre os que bebiam acima de 50g/dia de álcool. Na regressão logística, foram significativas as variáveis consumo de tabaco por mais de 20 anos $(O R=10,1)$, consumo de cigarros industrializados $(O R=4,8)$ e consumo de álcool acima de 50g/dia $(O R=2,9)$. Os doentes que fumavam 20 ou mais cigarros/dia adoeceram em média oito anos antes que os demais $(p=0,002)$. O consumo de álcool acima de 50g/dia não influenciou na idade de adoecimento $(p=0,78)$. Conclui-se que o tabagismo constitui fator de risco para o desenvolvimento da paracoccidioidomicose; o alcoolismo atuaria como um co-fator, associado ao tabagismo.

Palavras-chave Paracoccidioidomicose; Tabagismo; Alcoolismo; Fatores de Risco 


\section{Introdução}

O tabagismo é considerado a principal causa de morte evitável no mundo. Segundo estimativas da Organização Mundial da Saúde (OMS), existe cerca de 1,1 bilhão de fumantes no mundo, o que representa um terço da população mundial acima de quinze anos, dos quais cerca de 800 milhões estão nos países subdesenvolvidos. Estima-se que $47 \%$ dos homens e $12 \%$ das mulheres do mundo fumam, sendo $48 \%$ de homens e $7 \%$ de mulheres nos países subdesenvolvidos e $42 \%$ dos homens e $24 \%$ das mulheres nos países desenvolvidos (INCA, 1997).

No Brasil, 32,6\% da população adulta fumam, sendo 11,2 milhões de mulheres e 16,7 milhões de homens. A faixa de 20 a 49 anos de idade concentra a maioria dos fumantes. Em todas as faixas etárias há predomínio de fumantes do sexo masculino. O maior consumo está nas camadas sociais com menor poder aquisitivo e é proporcionalmente maior entre os moradores da zona rural (MS, 1998). Chaieb \& Castellarin (1998) encontraram maior prevalência de fumantes entre alcoolistas, que entre nãoalcoolistas, com tendência de os primeiros começarem a fumar mais cedo, além de fumarem por mais tempo e maior número de cigarros.

Da fumaça do cigarro, principal forma de consumo do tabaco, já foram identificadas 4.720 substâncias, que podem ser classificadas em 15 funções químicas diferentes. As principais são a nicotina e seus derivados, que causam a dependência química e também efeitos sobre os sistemas nervoso e circulatório; o monóxido de carbono, que altera o transporte de oxigênio no sangue para os tecidos; os compostos cancerígenos; as substâncias adicionadas no processo de cultivo do tabaco e na sua manufatura e finalmente as substâncias irritantes da mucosa respiratória, que levam ao desenvolvimento de bronquite e favorecem o desenvolvimento de infecções respiratórias (Rosemberg, 1987).

A acroleína e seus derivados, as mais importantes das substâncias irritativas da fumaça do tabaco, causam efeitos tanto imediatos, quanto tardios no aparelho respiratório. Irritação, inflamação, alergia, tosse, broncoconstrição e paralisação dos movimentos ciliares do epitélio respiratório (ciliostase) são efeitos imediatos encontrados. Estimulação de secreção de muco, perda dos cílios, outras alterações do epitélio dos brônquios, alteração na atividade enzimática e imunitária dos macrófagos alveolares, além de inflamação crônica brônquica e destruição alveolar são os efeitos tardios encontrados nos pulmões dos fumantes (Rosemberg, 1987).
O tabagismo está relacionado a uma maior morbimortalidade, e os fumantes adoecem 3,5 vezes mais que os não-fumantes. O tabaco é considerado fator de risco para cerca de 25 doenças, entre elas o câncer do lábio, cavidade oral, faringe, esôfago, pâncreas, laringe, pulmão, traquéia e brônquios, bexiga, pelve renal; doenças cardiovasculares; cerebrovasculares; doenças pulmonares obstrutivas crônicas; doença vascular periférica; baixo peso em recém-nascido; úlcera péptica; gravidez malsucedida; aumento da mortalidade infantil, incluindo a síndrome da morte súbita infantil. Entre as doenças infecciosas, a tuberculose e as infecções respiratórias baixas têm sido associadas com o hábito de fumar, em qualquer idade. Há evidências de que o tabaco afeta a imunidade humoral e a mediada por células, sendo fator de risco para doenças notadamente pulmonares, bacterianas e viróticas (INCA, 1997; Rosemberg, 1987, 1997). Entre 34.439 médicos ingleses que participaram de um estudo prospectivo sobre a mortalidade associada ao tabagismo, o risco de morrer por tuberculose pulmonar foi de 3,7 entre os tabagistas, quando comparados aos não-fumantes (Doll et al., 1994).

A paracoccidioidomicose é uma micose sistêmica, de evolução arrastada, descrita pela primeira vez por Adolpho Lutz, em 1908, no Estado de São Paulo, Brasil (Del Negro et al., 1982). O agente etiológico é o Paracoccidioides brasiliensis, um fungo assexuado e dimórfico, de crescimento lento em meios de cultura. No Brasil, há poucos relatos de isolamento do fungo de animais silvestres, como tatus (Bagagli et al., 1998), e do solo (Ferreira et al., 1990; Montenegro et al., 1996). Esta é a principal dificuldade encontrada no estudo de sua distribuição geográfica, sendo praticamente desconhecidos os fatores que favorecem seu desenvolvimento no meio ambiente.

Sabe-se, hoje, que a doença é endêmica em toda a América Latina, predominantemente nas regiões entre $20^{\circ}$ ao Norte e $35^{\circ}$ ao Sul do Equador, estando presente do México até a Argentina. As condições ambientais favoráveis para a sua presença são: temperaturas entre $10-28^{\circ} \mathrm{C}$; chuvas entre $500-2.500 \mathrm{~mm} /$ ano; clima temperado a quente, moderadamente úmido; abundantes florestas nativas; altitude variável, entre 47-1300m acima do nível do mar; abundantes cursos de água; invernos curtos; verões chuvosos e solo quase sempre ácido (Restrepo, 1985). Calle et al. (2001), em um estudo ecológico na Colômbia, encontraram associação entre uma maior incidência da doença nas regiões com altitude entre 1.000 a 1.499 metros acima do nível do mar, precipitações entre 2.000 a $2.999 \mathrm{~mm}$, 
presença de florestas úmidas e regiões de colheita de café e tabaco.

Na América Latina, o Brasil apresenta a maior estatística de casos, distribuídos principalmente em São Paulo, Rio de Janeiro, Minas Gerais, Paraná, Rio Grande do Sul, Goiás e Mato Grosso do Sul, registrando-se os números mais expressivos em São Paulo. Não sendo uma doença de notificação compulsória, não temos sua incidência real. Em zonas endêmicas, esta é estimada em um a três casos por 100 mil habitantes. A moléstia acomete preferencialmente trabalhadores que estão em contato com o solo, como os agricultores, e, dentre eles, é comum nos da lavoura do café. Também está presente entre trabalhadores da construção civil, que na maioria das vezes são oriundos da zona rural e do trabalho no campo (Martinez, 1996).

Em 54 inquéritos epidemiológicos com a paracoccidioidina, realizados em vários estados do Brasil, alguns na população geral, outros em crianças e escolares, a positividade do teste variou de $2 \%$ entre crianças de bairros pobres do Rio de Janeiro a $82 \%$ entre soldados em Cachoeira do Sul, Rio Grande do Sul (Fava \& Fava Netto, 1998).

No Estado do Espírito Santo, a incidência da moléstia parece relativamente elevada, a julgar pela freqüência com que os doentes buscam atendimento nos serviços médicos de referência. No entanto, existem poucos estudos sobre a epidemiologia da doença nesse Estado. Na zona rural do Município de Colatina, Espírito Santo, Vieira (1979) realizou inquérito com teste intradérmico da paracoccidioidina entre alunos e funcionários de um colégio agrícola e entre habitantes das cercanias deste, encontrando uma taxa de 20,05\% de reatores.

Quanto ao mecanismo da infecção humana, estudos clínicos e laboratoriais demonstram ser a via inalatória a porta de entrada do P. brasiliensis no organismo. Após atingir os pulmões, o fungo dissemina-se por via linfática, hematogênica, atingindo diversos órgãos, e por via canalicular, através da árvore respiratória. A evolução da infecção para doença depende da virulência do agente e da capacidade de resposta imunológica do hospedeiro, em especial da imunidade celular e do sistema fagocítico mononuclear (Ferreira, 1996; Montenegro \& Franco, 1994). Considerando os elevados índices de testes intradérmicos positivos em determinadas populações, a incidência relativamente baixa de casos clínicos da doença entre essas pessoas sugere que o fungo seja de baixa patogenia.

A doença é classificada clinicamente como regressiva, progressiva ou seqüelar. A forma progressiva pode ser aguda ou subaguda (tipo ju- venil) ou crônica (tipo adulto) (Mendes, 1996). A forma juvenil manifesta-se geralmente em pessoas com menos de trinta anos de idade, caracterizando-se principalmente pelo comprometimento de órgãos ricos em células do sistema fagocítico mononuclear, como os gânglios linfáticos, baço, fígado e medula óssea. Esta é a forma mais grave da doença que, em crianças abaixo de dez anos de idade, acomete indistintamente ambos os sexos e, a partir da segunda década de vida, passa a predominar no sexo masculino.

A forma crônica da moléstia manifesta-se quase sempre a partir da quarta década, acometendo principalmente os pulmões, a mucosa oral e o trato respiratório superior, além da pele. Predomina de forma quase absoluta no sexo masculino, suspeitando-se que, além de fatores hormonais (Yasuda \& Restrepo, 1996), participem no desenvolvimento da doença fatores de risco externos, agindo diretamente sobre o trato respiratório, ou atuando na imunidade mediada por células. Mesmo com o tratamento adequado, a forma crônica pode deixar, como seqüela, grave disfunção respiratória, principal manifestação da forma seqüelar da doença.

Entre as diversas vertentes dos estudos epidemiológicos sobre a paracoccidioidomicose, uma das linhas de pesquisa busca identificar fatores que levam o indivíduo infectado a manifestar a doença. Na forma crônica, o período decorrido entre a infecção e o início de manifestações clínicas pode ser muito longo. Pessoas infectadas, morando em áreas indenes, podem adoecer até trinta anos depois de terem deixado a área endêmica (Restrepo, 1990). Os primeiros sintomas surgem quase sempre na faixa etária dos 30 aos 50 anos de idade, no sexo masculino.

Entre os fatores desencadeantes da doença, têm sido citados o alcoolismo e a desnutrição. Em pacientes portadores de doenças imunossupressoras, como a AIDS, o fungo pode se comportar como um agente oportunista (Martinez, 1996).

Murray et al. (1974), em relato de um paciente que desenvolveu a forma disseminada, referem que este fumava um maço de cigarros por dia. Em uma série de vinte casos com a forma crônica, todos os pacientes eram fumantes. (Lemle et al., 1983). Outros autores também relatam a presença do tabagismo entre os pacientes com a forma crônica da doença (Washburn \& Bennett, 1986). Oliveira et al. (1997), em estudo de 54 casos de paracoccidioidomicose oral, relatam que dois terços dos pacientes eram tabagistas. Há referência ainda do uso de álcool e fumo por pacientes com manifestações laríngeas na paracoccidioidomicose (Sant'Anna et 
al., 1999). Apesar das menções feitas por diversos autores, em revisão recente da literatura não encontramos qualquer estudo controlado associando o tabagismo com a paracoccidioidomicose.

No Serviço de Doenças Infecciosas do Hospital Universitário onde foi realizado este estudo, são atendidos muitos pacientes portadores da doença. Em estudo prévio não controlado, observou-se que a maioria dos pacientes com a forma crônica da paracoccidioidomicose apresentava tabagismo associado. Nesse sentido, o presente trabalho teve como objetivo investigar a influência do tabagismo como fator de risco no desenvolvimento da forma crônica desta doença. Como já existe na literatura trabalho demonstrando a associação da paracoccidioidomicose com alcoolismo (Martinez \& Moya, 1992), esta variável também foi considerada nesta investigação.

\section{Material e métodos}

Com base no atendimento da demanda de pacientes que procuravam o Serviço de Doenças Infecciosas do Hospital Universitário Cassiano Antonio Moraes da Universidade Federal do Espírito Santo, no período de julho de 1999 a julho de 2000, foram selecionadas pessoas portadoras da forma crônica da paracoccidioidomicose, para compor o grupo dos casos da doença, em um estudo do tipo caso-controle. Foram adotados os seguintes critérios para inclusão dos pacientes (casos) no estudo: diagnóstico confirmado de paracoccidioidomicose pela demonstração do agente etiológico em exame histopatológico, raspado de lesão ou exame de escarro, idade superior a trinta anos, sem adenomegalias sugestivas da forma juvenil aguda e ausência de co-infecção pelo vírus HIV.

Para compor o grupo controle foram programadas visitas a 34 localidades rurais, distribuídas em 23 municípios do Estado do Espírito Santo, constando estes da relação dos mais citados, como locais de nascimento, residência e procedência imediata dos doentes. Dada a dificuldade para se determinar a renda entre os trabalhadores rurais, e considerando que os doentes eram quase sempre pessoas de padrão sócio-econômico mais baixo, o grupo controle foi composto de meeiros, diaristas, empregados rurais e pequenos proprietários, que tivessem pelo menos cinco anos de atividade no meio rural. Na tentativa de melhor atender aos requisitos acima, selecionava-se cerca de $20 \%$ das habitações de cada localidade, procurandose visitar as casas com padrão de construção mais simples, em busca de pessoas que preenchessem os demais critérios para composição do grupo controle como se descreve a seguir: moradores nas localidades, sem limite superior de idade e com limite inferior de 32 anos, correspondente à idade do doente mais jovem, quando do início dos sintomas, sem história de doença crônica compatível com paracoccidioidomicose, comprometendo pulmões, pele ou mucosa. O pareamento em relação ao sexo manteve também a mesma proporção de controles para cada caso. Evitou-se sempre entrevistar as pessoas fora de suas casas, em bares, igrejas e outros locais que pudessem causar viés de seleção dos controles.

Para a coleta de dados foi utilizado um questionário padronizado com questões sobre dados sócio-demográficos, ocupação, local onde nasceu, morou e trabalhou. Em relação aos hábitos de vida, anotaram-se dados sobre o uso de álcool e tabaco, idade de início, tempo de consumo, periodicidade e quantidade consumida. Para o cálculo da quantidade de álcool consumido, foram avaliados os teores médios do produto nos diversos tipos de bebidas, como se segue: aguardente de cana $=40 \%$; cerveja $=5 \%$; vinho $=12 \%$; outros destilados $=55 \%$. A intensidade do alcoolismo foi padronizada, calculando-se a média de consumo diário em gramas. Para aqueles que consumiam o produto somente nos fins de semana, a média diária representou o total de álcool consumido por semana, dividido por sete. Foram considerados ex-alcoolistas os que deixaram de consumir o produto há pelo menos cinco anos. Em relação ao tabaco, a intensidade de consumo diário representou a soma dos cigarros consumidos por dia, considerando-se cada cigarro de palha equivalente a cinco industrializados (Suzuki, et al., 1994). Da mesma forma, foram considerados ex-fumantes aqueles que pararam de fumar há pelo menos cinco anos.

Na ficha dos doentes foram anotados também o critério de diagnóstico, a forma clínica da doença e a idade de adoecimento. Ainda no grupo dos doentes, a classificação de ex-fumante e ex-alcoolista referiu-se ao abandono do fumo e do álcool pelo menos cinco anos antes do aparecimento dos primeiros sintomas.

Para o processamento e análise estatística dos dados, utilizou-se o programa SPSS versão 8.0 para Windows 95 .

Inicialmente foi aplicado o teste de hipóteses qui-quadrado para determinar as associações estatisticamente significativas entre as variáveis estudadas. Foi também calculado o odds ratio para as variáveis, considerando como referência a categoria ausência do fator em estudo, 
além do intervalo de confiança de $95 \%$. O teste de hipóteses $\mathrm{t}$, para amostras independentes, foi utilizado para comparar as médias das variáveis estudadas nos grupos caso e controle e também para comparar as médias de idade de adoecimento nos subgrupos dos doentes, divididos de acordo com a maior ou menor exposição aos supostos fatores de risco. O teste não paramétrico de Levene foi utilizado para testar se as variâncias dos dois grupos eram iguais, pressuposto básico para a aplicação do teste t. Em todos os testes, o nível de significância adotado foi $\mathrm{p}<0,05$.

Para a análise por meio da regressão logística, foram utilizadas como variáveis independentes aquelas que se mostraram estatisticamente significativas na análise univariada e, possivelmente, relacionadas à doença, sendo dicotomizadas da seguinte maneira: SIM, para a presença do fator, e NÃO, para a ausência do mesmo. Utilizou-se o método forward stepwise LR, com níveis de significância de $10 \%$ para inclusão e $15 \%$ para exclusão das variáveis.

Quanto aos aspectos éticos, todas as informações do presente estudo foram de caráter confidencial, não sendo divulgado o nome dos participantes a pessoas que não estivessem diretamente ligadas ao estudo. Os candidatos eram informados sobre o tipo e a finalidade do estudo, bem como a garantia da confidencialidade das informações. Após o esclarecimento de dúvidas porventura existentes, os mesmos eram convidados a responder às perguntas contidas no questionário. Havendo o consentimento verbal do candidato, iniciava-se a entrevista. Este estudo foi aprovado pelo Comitê de Ética em Pesquisa do Centro Biomédico da Universidade Federal do Espírito Santo, conforme parecer do processo 0987/01-47.

\section{Resultados}

Dos 81 pacientes com paracoccidioidomicose atendidos no Serviço de Doenças Infecciosas do Hospital Universitário Cassiano Antonio Moraes, no período de julho de 1999 a julho de 2000, nove foram excluídos por apresentarem quadro clínico compatível com a forma aguda e duas pacientes, por apresentarem co-infecção com HIV.

O grupo que preencheu os critérios para inclusão no estudo foi composto por setenta pacientes com a forma crônica da paracoccidioidomicose. A média de idade dos doentes foi de 52,6 anos (DP $\pm 10,5$ anos) e mediana de 51,5 anos, variando de 32 a 76 anos. Sessenta e nove $(98,6 \%)$ eram do sexo masculino e uma $(1,4 \%)$ do sexo feminino. Sessenta e cinco $(92,8 \%)$ fu- mavam no momento em que adoeceram e cinco $(7,2 \%)$ haviam parado de fumar há pelo menos cinco anos antes do adoecimento. Quanto ao uso de bebidas alcoólicas, $55(78,6 \%)$ relatavam o consumo, 11 (15,7\%) haviam parado de beber e quatro $(5,7 \%)$ negavam o uso de álcool. Em 54 pacientes $(77,2 \%)$, a radiografia de tórax evidenciava a presença de lesões pulmonares, e em 35 (50\%) estas estavam associadas a lesões mucosas; em três $(4,3 \%)$, a lesões cutâneas e em sete $(10,0 \%)$, a lesões cutâneo-mucosas.

Entre os 180 indivíduos controles, a média de idade foi de 55,6 anos (DP $\pm 14,0$ anos), e mediana de 54 anos, variando de 33 a 92 anos. Cento e setenta e sete $(98,3 \%)$ eram do sexo masculino e três $(1,7 \%)$ do sexo feminino. Oitenta e cinco indivíduos $(47,2 \%)$ eram fumantes, 47 (26, $1 \%)$ ex-fumantes e $48(26,7 \%)$ não fumantes. Em relação ao alcoolismo, $123(68,3 \%)$ relatavam uso de bebidas alcoólicas, $37(20,5 \%)$ haviam parado de beber e vinte $(11,1 \%)$ não usavam bebidas alcoólicas.

Na Tabela 1, são apresentados os resultados comparativos das variáveis de consumo de tabaco e álcool estudado nos grupos caso e controle.

Ao compararmos a média das variáveis medidas nos casos e controles utilizando o teste $t$, os resultados indicam a existência de diferença na idade em que começou a fumar, no tempo de tabagismo e no número de cigarros fumados ao dia (Tabela 2).

Na regressão logística, as variáveis utilizadas foram: Atualmente fuma? Fuma há mais de vinte anos? Fuma cigarro industrializado? Fuma cigarros de palha? Fuma ambos os tipos de cigarros? Fuma vinte ou mais cigarros por dia? Bebe mais de 50g/dia de álcool? Bebe cachaça?

Entre os doentes, os tabagistas por mais de vinte anos mostraram maiores chances de desenvolver a doença, OR = 10,1 (IC 95\%: 2,2-45,8), seguido pelo consumo de cigarros industrializados, OR = 4,8 (IC 95\%: 2,2-10,7); porém, o consumo de $>50 \mathrm{~g} /$ dia de álcool mostrou menor chance para adoecer, OR = 2,9 (IC 95\%: 1,5-5,5), (Tabela 3).

A comparação da intensidade de consumo do tabaco e do álcool, em relação à média de idade de adoecimento, (Tabela 4) mostra que, no grupo dos fumantes de vinte ou mais cigarros/dia, a média de idade de adoecimento foi de 46,1 anos, enquanto, no segundo grupo, a média foi de 54 anos de idade. A diferença mostrou-se significativa ( $\mathrm{p}=0,002$ ), indicando que os indivíduos que fumavam vinte ou mais cigarros/dia adoeceram em média oito anos antes que os demais. O mesmo tipo de análise foi feito em relação ao consumo de álcool, comparando os que bebiam $>50 \mathrm{~g} /$ dia com os demais. 
Tabela 1

Resultado comparativo dos grupos caso e controle para as diversas variáveis estudadas, valores do qui-quadrado e p-valor, odds ratio com o intervalo de confiança.

\begin{tabular}{|c|c|c|c|c|c|c|}
\hline Variável & Caso n & Controle $n$ & $\chi^{2}$ & p-valor* & Odds ratio & $\begin{array}{c}\text { Intervalo de } \\
\text { confiança } 95 \%\end{array}$ \\
\hline Fumo & & & 43,7 & 0,000 & 14,5 & $5,6-37,7$ \\
\hline Não/Ex-fumante & 5 & 95 & & & & \\
\hline Sim & 65 & 85 & & & & \\
\hline Tipo de cigarro & & & 54,3 & 0,000 & & \\
\hline Não fuma** & 5 & 95 & & & 1 & - \\
\hline Industrializado & 11 & 14 & & & 14,9 & $4,5-49,4$ \\
\hline Palha & 5 & 21 & & & 4,5 & $1,2-17,0$ \\
\hline Ambos & 49 & 49 & & & 19,0 & $7,1-50,8$ \\
\hline Outros tipos & 0 & 1 & & & 1,0 & $1,0-1,0$ \\
\hline Álcool & & & 28,5 & 0,000 & & \\
\hline Não bebe $e^{\star \star}$ & 4 & 20 & & & 1 & - \\
\hline Parou há 5 anos & 11 & 37 & & & 1,5 & $0,4-5,3$ \\
\hline Socialmente & 4 & 31 & & & 0,6 & $0,1-2,9$ \\
\hline$<50 \mathrm{~g} \mathrm{dia} / \mathrm{fse}^{\star \star \star}$ & 6 & 32 & & & 0,9 & $0,2-3,7$ \\
\hline$>50 \mathrm{~g} \mathrm{dia} / \mathrm{fse}^{\star \star \star}$ & 11 & 6 & & & 9,2 & $2,1-39,6$ \\
\hline$<50 \mathrm{~g}$ dia & 3 & 11 & & & 1,4 & $0,2-7,2$ \\
\hline$>50 \mathrm{~g} \mathrm{dia}$ & 31 & 43 & & & 3,6 & $1,1-11,6$ \\
\hline Tipo de bebida & & & 17,0 & 0,004 & & \\
\hline Não bebe ${ }^{\star \star}$ & 15 & 57 & & & 1 & - \\
\hline Destilados & 1 & 0 & & & 0,9 & $0,8-1,1$ \\
\hline Cachaça & 51 & 93 & & & 2,1 & $1,1-4,0$ \\
\hline Vinho & 0 & 3 & & & 1,0 & $1,0-1,1$ \\
\hline Cerveja & 1 & 24 & & & 0,2 & $0,0-1,3$ \\
\hline
\end{tabular}

* em itálico os valores estatisticamente significativos.

** categoria considerada referência para o cálculo do odds ratio.

*** média diária do total de álcool consumido no fim de semana.

Tabela 2

Média e desvio padrão (DP) das variáveis dos casos e controles e teste t com o p valor.

\begin{tabular}{lcccc}
\hline Variáveis & $\begin{array}{c}\text { Caso } \mathbf{n}=70 \\
\text { Média }( \pm \text { DP) }\end{array}$ & $\begin{array}{c}\text { Controle } \mathbf{n}=180 \\
\text { Média ( } \pm \text { DP) }\end{array}$ & Teste t $\mathbf{p ~ v a l o r}$ \\
\hline Idade em anos & $52,6( \pm 10,5)$ & $55,6( \pm 14,0)$ & $-1,8$ & 0,061 \\
Idade em anos em que começou fumar & $13,5( \pm 3,7)$ & $10,9( \pm 8,0)$ & 3,5 & 0,001 \\
Tempo em anos que fuma & $38,2( \pm 10,6)$ & $24,3( \pm 21,5)$ & 6,8 & 0,000 \\
Número de cigarros/dia & $18,1( \pm 12,5)$ & $6,6( \pm 9,8)$ & 6,9 & 0,000 \\
Tempo em anos que parou de fumar & $11,0( \pm 3,2)$ & $20,7( \pm 11,4)$ & $-1,6$ & 0,113 \\
Tempo em anos que parou de beber & $13,0( \pm 5,3)$ & $18,5( \pm 15,5)$ & $-2,0$ & 0,057 \\
Idade em anos em que começou a beber & $16,0( \pm 33,0)$ & $16,2( \pm 15,2)$ & $-1,9$ & 0,850 \\
Tempo em anos que bebeu álcool & $30,3( \pm 29,7)$ & $28,1( \pm 20,51)$ & 0,8 & 0,396 \\
\hline
\end{tabular}


No primeiro grupo, a média de idade de adoecimento foi de 49,3 anos, enquanto no segundo a média foi de cinqüenta anos. A diferença não foi significativa ( $p=0,780$ ), indicando que a intensidade do consumo de álcool não influenciou na idade de adoecimento.

\section{Discussão}

Diversos autores relatam a presença do tabagismo entre portadores da forma crônica da paracoccidioidomicose em séries descritivas de casos (Bisinelli et al., 2001; Lemle et al., 1983; Oliveira et al., 1997; Sant'Anna et al., 1999; Washburn \& Bennett, 1986). Também Del Negro (1986), em sua experiência, sugere que o tabagismo possa influenciar na recidiva de lesões pulmonares em pacientes considerados clinicamente curados. Por outro lado, Wanke (1976) não encontrou influência do fumo de cigarros de palha de milho e cachimbo favorecendo a infecção pelo P. brasiliensis. Quanto ao alcoolismo, Martinez \& Moya (1992), em estudo casocontrole, demonstraram associação entre o consumo excessivo de álcool, em especial a aguardente de cana, e a forma crônica da doença.

Neste trabalho, a análise estatística mostrou que os doentes consumiam mais bebida alcoólica que os sadios e que a chance de adoecer era maior naqueles que consumiam mais de $50 \mathrm{~g}$ de álcool diariamente ou nos fins de semana e entre os consumidores de cachaça. Neste ponto, os resultados deste estudo concordam perfeitamente com as observações de Martinez \& Moya (1992). No entanto, os doentes também fumavam mais que os controles, sendo maior a chance de adoecer entre aqueles que fumavam cigarros de palha e industrializados juntos (Tabela 1). Os doentes, em média, fumavam há mais tempo maior número de cigarros, enquanto os controles haviam parado de fumar e beber bebidas alcoólicas há mais tempo. Os casos também, em média, consumiam álcool há mais tempo que os controles. Os doentes, em média, fumavam há mais tempo maior número de cigarros, enquanto os controles começaram a fumar mais precocemente (Tabela 2).

O consumo de tabaco por mais de vinte anos, o consumo de cigarros industrializados e o consumo de $>50 \mathrm{~g} /$ dia de álcool estão associados à maior chance de adoecimento, aumentando nas variáveis relacionadas ao tabagismo, caso em que estas chances são respectivamente 10,1 e 4,8 maiores. Já para o consumo de álcool, esta chance é 2,9 maior (Tabela 3).

No grupo dos doentes, os consumidores de vinte ou mais cigarros/dia adoeceram em mé-
Tabela 3

Variáveis associadas à forma crônica da paracoccidioidomicose com os respectivos odds ratio e Intervalo de confiança de $95 \%$.

\begin{tabular}{lcc}
\hline Variável & Odds ratio & Intervalo de confiança (95\%) \\
\hline$\geq 20$ anos de fumo & 10,1 & $2,2-45,8$ \\
Cigarro industrializado & 4,8 & $2,2-10,7$ \\
$>50 \mathrm{~g}$ de álcool/dia & 2,9 & $1,5-5,5$ \\
\hline
\end{tabular}

Tabela 4

Comparação da média de idade de adoecimento em relação à intensidade

do consumo de tabaco e álcool entre os pacientes com a forma crônica

de paracoccidioidomicose.

\begin{tabular}{lcccc}
\hline Grupos & No de doentes & $\begin{array}{l}\text { Média de idade } \\
\text { de adoecimento } \\
\text { (anos) }\end{array}$ & Teste t & p valor \\
\hline$\geq 20$ cigarros dia & 38 & 46,1 & & \\
$<20$ cigarros dia & 32 & 54,0 & 3,3 & 0,002 \\
$>50 \mathrm{~g} /$ dia de álcool & 31 & 49,3 & & 0,780 \\
$<50 \mathrm{~g} /$ dia de álcool & 39 & 50,0 & $-2,8$ & \\
\hline
\end{tabular}

dia oito anos antes que os consumidores de menos de vinte cigarros/dia, enquanto a maior intensidade do alcoolismo não influenciou na idade de adoecimento (Tabela 4).

Com base nos resultados obtidos, concluise que o tabagismo constitui um importante fator de risco para o desenvolvimento da forma crônica da paracoccidioidomicose. Quanto ao alcoolismo, há evidências que atue como um co-fator de risco, associado ao tabagismo.

Para explicar o mecanismo pelo qual o tabaco atuaria, poderíamos sugerir a ação das substâncias irritantes presentes na fumaça do tabaco e inaladas pelos fumantes, como a acroleína e seus derivados, com seus efeitos de irritação, inflamação, alergia, broncoconstrição, ciliostase, até perda dos cílios, aumento da secreção de muco, alteração da atividade enzimática e imunitária dos macrófagos que levam ao desenvolvimento de inflamação brônquica crônica e destruição alveolar favorecendo o desenvolvimento de infecções respiratórias (Rosemberg, 1987). Além da conhecida proteção do hormônio feminino (Yasuda \& Restrepo, 1996), outra questão a ser levantada seria a de que a maior prevalência da forma crônica da doença no sexo masculino poderia estar relacionada ao fato 
de que a prevalência de tabagismo é maior no homem (INCA, 1997; MS, 1998), já que até a puberdade não há diferença da incidência da doença entre os sexos (Martinez, 1996) nem tampouco da prevalência da infecção, medida por meio de testes intradérmicos com paracoccidioidina (Restrepo, 1990; Wanke, 1976).

Tendo em vista a dimensão e a gravidade da epidemia do tabagismo, é de grande relevância que novos estudos sejam realizados e que seus resultados sejam divulgados não só para a comunidade científica, mas também para a população em geral, pois parte do controle do ta-

\section{Referências}

BAGAGLI, E.; SANO, A.; COELHO, K. I.; ALQUATI, S.; MIYAJI, M.; CAMARGO, Z. P.; GOMES, G. M.; FRANCO, M. \& MONTENEGRO, M. R., 1998. Isolation of Paracoccidioides brasiliensis from armadillos (Dasypus noveminctus) captured in an endemic área of paracoccidioidomycosis. American Journal of Tropical Medicine and Hygiene, 58:505-512.

BISINELLI, J. C.; TELLES, F. Q.; SOBRINHO, J. A. \& RAPOPORT, A., 2001. Manifestações estomatológicas da paracoccidioidomicose. Revista Brasileira de Otorrinolaringologia, 67:683-687.

CALLE, D.; ROSERO, D. S.; OROZCO, L. C.; CAMARGO, D.; CASTANEDA, E. \& RESTREPO, A., 2001. Paracoccidioidomycosis in Colombia: An ecological study. Epidemiology and Infection, 126:309-315

CHAIEB, J. A. \& CASTELLARIN, C., 1998. Associação tabagismo-alcoolismo: Introdução às grandes dependências humanas. Revista de Saúde Pública, 32:246-254.

DEL NEGRO, G.; LACAZ, C. S. \& FIORILLO, A. M., 1982. Paracoccidioidomicose. Blastomicose Sul-Americana. São Paulo: Editora Sarvier/Edusp.

DEL NEGRO, G., 1986. Paracoccidioidomicose. II - Tratamento: Noções práticas. Jornal de Pneumologia, 21:57-60.

DOLL, R.; PETO, R.; WHEATLEY, K.; GRAY, R. \& SUTHERLAND, I., 1994. Mortality in relation to smoking: 40 years' observations on male British doctors. BMJ, 309:901-911.

FAVA, S. \& FAVA NETTO, C., 1998. Epidemiologic surveys of Histoplamin and Paracoccidioidin sensitivity in Brazil. Revista do Instituto de Medicina Tropical de São Paulo, 40:155-164.

FERREIRA, M. S., 1996. Blastomicose Sul-Americana (Paracoccidioidomicose): Imunopatogênese e patologia. In: Tratado de Infectologia (R. Veronesi \& R. Focaccia, org.), pp. 1083-1087, São Paulo: Editora Atheneu.

FERREIRA, M. S.; FREITAS, M. H.; LACAZ, C. S.; DEL NEGRO, G. M. B.; MELO, N. T.; GARCIA, N. M.; ASSIS, C. M.; SALEBIAN, A. \& HEINS-VACCARI, E. M., 1990. Isolation and characterization of a Paracoccidioides brasiliensis strain from a dogfood probably contaminated with soil in Uberlândia, bagismo está na divulgação de novos conhecimentos sobre o assunto (Prabhat \& Chaloupka, 2000). Também é de relevância um melhor conhecimento das conseqüências do consumo do tabaco na nossa população, dentro do contexto sócio-econômico e cultural próprio. Por outro lado, o conhecimento dessa provável associação encontrada é mais uma ferramenta na prevenção e tratamento da paracoccidioidomicose, inclusive com o incentivo e oferta de tratamento específico para a dependência à nicotina aos pacientes com paracoccidioidomicose, além de ações educativas em saúde.
Brazil. Journal of Medical and Veterinary Mycology, 28:253.

INCA (Instituto Nacional de Câncer), 1997. Manual de Orientação Dia Mundial sem Tabaco, 31 de Maio de 1997. Rio de Janeiro: INCA.

LEMLE, A.; WANKE, B. \& MANDEL, M. B., 1983. Pulmonary localization of Paracoccidioidomycosis: Lung function studies before and after treatment. Revista do Instituto de Medicina Tropical de São Paulo, 25:73-78.

MARTINEZ, R., 1996. Blastomicose Sul-Americana (Paracoccidioidomicose): Etioepidemiologia e ecologia. In: Tratado de Infectologia (R. Veronesi \& R. Focaccia, org.), pp. 1081-1083, São Paulo: Editora Atheneu.

MARTINEZ, R. \& MOYA, M. J., 1992. Associação entre paracoccidioidomicose e alcoolismo. Revista de Saúde Pública, 26:12-16.

MENDES, R. P., 1996. Blastomicose Sul-Americana (Paracoccidioidomicose): Quadro clínico. In: Tratado de Infectologia (R. Veronesi \& R. Focaccia, org.), pp. 1087-1098, São Paulo: Editora Atheneu.

MONTENEGRO, M. R. \& FRANCO, M., 1994. Pathology. In: Paracoccidioidomycosis (M. Franco, C. S. Lacaz, A. Restrepo-Moreno \& G. Del Negro, ed.), pp. 131-150, Boca Raton: CRC Press.

MONTENEGRO, M. R.; MIYAJI, M.; FRANCO, M.; NISHIMURA, K.; COELHO, K. I.; HORIE, Y.; MENDES, R. P.; SANO, A.; FUKUSHIMA, K. \& FECCHIO, D., 1996. Isolation of fungi from nature in region of Botucatu, state of São Paulo, Brazil, an endemic area of paracoccidioidomycosis. $\mathrm{Me}$ mórias do Instituto Oswaldo Cruz, 91:665-670.

MS (Ministério da Saúde), 1998. Falando sobre Tabagismo. Rio de Janeiro: Instituto Nacional de Câncer.

MURRAY, H. W.; LITTMAN, M. L. \& ROBERTS, R. B., 1974. Disseminated Paracoccidioidomycosis (South American Blatomycosis) in the United States. American Journal of Medicine, 56:209-220.

OLIVEIRA, P. T.; YURGEL, L. S.; LORANDI, C. S. \& MORAES, A. C. S., 1997. Estudo de 54 casos de paracoccidioidomicose diagnosticados no Serviço de Estomatologia do Hospital São Lucas da PUCRS. Revista Médica da PUCRS, 7:161-166. 
PRABHAT, J. \& CHALOUPKA, F. J., 2000. A Epidemia do Tabagismo: Os Governos e os Aspectos Econômicos do Controle do Tabaco. Washington, DC: World Bank.

RESTREPO, A. M., 1985. The ecology of Paracoccidioides brasiliensis: A puzzle still unsolved. Journal of Medical and Veterinary Mycology, 23:323-334.

RESTREPO, A. M., 1990. Paracoccidioides brasiliensis. In: Principles and Practice of Infectious Diseases (G. L. Mandell, R. G. Douglas \& J. E. Bennet, ed.), pp. 2028-2031, 3rd Ed., New York: Churchil Livingstone Inc.

ROSEMBERG, J., 1987. Tabagismo: Sério Problema de Saúde Pública. 2a Ed. São Paulo: Editora Almed.

ROSEMBERG, J., 1997. Tabagismo e tuberculose. Boletim de Pneumologia Sanitária, 5:7-24.

SANT'ANNA, G. D.; MAURI, M.; ARRARTE, J. L. \& CAMARGO Jr., H., 1999. Laringeal manifestations of paracoccidioidomycosis. Archives of Otolaryngology - Head and Neck Surgery, 125:1375-1378.

SUZUKI, I.; HAMADA, G. S.; ZAMBONI, M. M.; CORDEIRO, P. B.; WATANABE, S. \& TSUGANE, S., 1994. Risk factors for lung cancer in Rio de Janeiro, Brazil: A case-control study. Lung Cancer, 11:179190.
VIEIRA, N. F. R., 1979. Contribuição para o Estudo da Paracoccidioidomicose Infecção no Estado do Espírito Santo. Provas Intradérmicas à Paracoccidioidina, Histoplasmina e PPD. Dissertação de Mestrado, Belo Horizonte: Curso de Pós-Graduação em Medicina Tropical, Universidade Federal de Minas Gerais.

WANKE, B., 1976. Paracoccidioidomicose: Inquérito Intradérmico com Paracoccidioidina em Zona Urbana do Município do Rio de Janeiro. Dissertação de Mestrado, Rio de Janeiro: Faculdade de Medicina, Universidade Federal do Rio de Janeiro.

WASHBURN, R. G. \& BENNETT, J. E., 1986. Paracoccidioidomycosis case report: Cure with amphotericin B and triple sulfa. Journal of Medical and Veterinary Mycology, 24:235-237.

YASUDA, M. A. S. \& RESTREPO, A. M., 1996. Imunologia das micoses. In: Tratado de Infectologia (R. Veronesi \& R. Focaccia, org.), pp. 1058-1080, São Paulo: Editora Atheneu.

Recebido em 26 de setembro de 2001

Versão final reapresentada em 22 de fevereiro de 2002 Aprovado em 23 de agosto de 2002 